\title{
Simple random-interval generation reveals the irresistibly periodic structure of perceived time
}

\author{
J. Eric T. Taylor ${ }^{1}$. Jessica A. Grahn ${ }^{1}$ \\ Published online: 28 May 2019 \\ (C) The Psychonomic Society, Inc. 2019
}

\begin{abstract}
Whole-integer ratios in musical rhythm are culturally universal. The reliable periodicity of rhythm inspired us to determine whether time perception, which is foundational to and inherently less structured than rhythm, is subject to similar biases. We created a random-interval generation task that exploits the nonrandom tendencies in perception and action in order to uncover the structural biases underlying temporal duration perception. Participants listened to and watched an audiovisual suprasecond temporal cue and were asked to subdivide it as randomly as possible in a prescribed number of responses. The results showed that the subdivision probability distributions were distinctly nonrandom, and closely resembled multimodal distributions with a number of equally spaced, symmetrical peaks equal to the number of subdivisions required. These patterns were thus highly periodic and isochronous, despite explicit instructions to act as randomly as possible. We interpreted this bias as an organizing heuristic that divides perceived time into smaller, equal-duration chunks in order to facilitate representation.
\end{abstract}

Keywords Temporal processing $\cdot$ Music cognition $\cdot$ Sound recognition

Universal features in musical rhythm are rare. Chief among them is an isochronous, metronomic beat that scaffolds timing into a metrical hierarchy of simple, small-integer subdivisions (Savage, Brown, Sakai, \& Currie, 2015). In this article, we ask whether the tendency toward periodic representations in rhythm also exists in the perception of time. We asked participants to randomly subdivide temporal intervals that contained no periodic or sequential information. (Indeed, hardly any information at all!) Unsurprisingly, we found that they did not perform randomly; surprisingly, their nonrandomness conformed to a periodic structure, despite the stimuli being amusical and arrhythmic.

Random-generation tasks have a long history of use in psychology to sound the depth of executive processing (Brugger, 1997). The most common implementation is random number generation, in which participants overrepresent certain digits or sequences and perform more randomly with the availability of executive resources. Less common are

J. Eric T. Taylor Jtay157@uwo.ca

1 The Brain \& Mind Institute, University of Western Ontario, London, Ontario, Canada paradigms that exploit our failures at randomness in order to reveal latent biases in cognition. For example, attempts to generate random sequences are biased by the direction people turn their head or move their eyes (Loetscher \& Brugger, 2007; Loetscher, Schwarz, Schubiger, \& Brugger, 2008), in a manner consistent with the mental number line (leftwardsmall, rightward-large). In another study, crowd-level data from a thousand people who randomly touched line drawings of shapes revealed a medial axis of symmetry, which is a covert shape perception strategy employed by the visual system (Firestone \& Scholl, 2014). In these examples, the human failure at randomness reveals biases in perception and representation. Here, we applied a similar logic to the perception of time.

Our approach was to provide participants with a minimally structured stimulus - a uniform interval of time - and an unconstrained task - to divide the interval as randomly or whimsically ("whenever you feel like it") as possible. Nonrandom patterns in the task would indicate biases in the representation of temporal duration (Vandierendonck, 2000). This logic recalls the foundational work by Fraisse (1946), who showed that reproduction of multiple uneven interval durations skews toward equality in an effort to reduce the cognitive load of multiple internal interval representations (Repp, London, \& Keller, 2011). More recently, it has been shown that 
participants bias their reproductions of random sequences of intervals toward integer-ratio relationships over multiple iterations (Jacoby \& McDermott, 2017; Ravignani, Delgado, \& Kirby, 2016). These studies demonstrate the human tendency to impose simple-integer ratios onto nonmetric or random temporal sequences. They also uncover the preferred temporal ratios when information is already present in the stimulus, in the form of subdivisions. Unlike the aforementioned studies, in which the stimuli contained sequential information (and therefore preexisting subintervals), the stimuli in the present study were inherently nonsequential and possessed no temporal structure beyond their singular duration. An inclination toward structured subdivisions would therefore be indicative of a powerful bias toward a periodic representation of temporal intervals.

\section{Method}

\section{Participants}

Fifty-five undergraduate students (36 female, 19 male; mean age $=19$ years) participated in exchange for course credit. All participants were naïve to the purpose of the experiment and provided informed consent. Forty-three of the participants reported having some musical training (mean $=4.36$ years, $S D=$ 3.83 ), although only eight participants reported practicing or performing regularly at the time of the experiment. Nineteen participants reported that English was not their native language, although all but one participant reported being fluent in English.

\section{Apparatus}

All data was collected using a Dell laptop running Matlab with the Psychtoolbox extension (Brainard, 1997). Auditory stimuli were presented using SONY over-ear headphones.

\section{Procedure}

Participants were instructed that the experiment would require that they press a button during an interval of sound. On every trial, they would hear a tone and see a progress bar fill up over the course of the interval. They would see and hear this stimulus twice, to familiarize themselves with the duration. On the third exposure, they would be cued to make one, two, or three responses during the interval (see Fig. 1). Depending on the block, participants were instructed to distribute these responses "as randomly as possible" or to make responses "whenever you feel like it." Participants were also instructed that in a random distribution of responses, all response times are equally likely, whereas responding at their whim had no prescribed order. The order of these blocks was counterbalanced by participant.

As we described above, the interval stimulus had auditory and visual components. This dual-modal stimulus was designed to increase the participant's ability to encode the duration accurately, as compared to a unimodal stimulus (Repp \& Penel, 2002; Thompson \& Paivio, 1994). The auditory component was a $500-\mathrm{Hz}$ sine wave at a comfortable volume set by the participant. The visual component was a horizontal loading bar that filled up over the course of the interval, from left to right. The color and location of the loading bar was randomly determined for every trial, to disrupt the sense of object continuity between trials. The RGB indices of the color were randomly determined to be between 100 and 220. The location of the stimulus was horizontally and vertically central, with a random jitter in both directions on every trial. The interval was 2,3 , or $4 \mathrm{~s}$ in length, randomized and balanced within a block. The number of responses required was one, two, or three, randomized and balanced within a block. There were eight repetitions of every combination of interval duration and response number, for 72 trials per block and 144 trials total. This yielded 15,480 observations in order to approximate the probability distribution functions for temporal subdivision. In comparison, Firestone and Scholl (2014) were able to achieve satisfactory shape perception distributions in experiments with 400 or fewer observations (one observations per person).

Throughout the trial, text appeared below the stimulus reminding participants of the instruction for that block and the cued number of responses for the third exposure. After the familiarization, participants were instructed to press the space bar when they were ready for the third exposure. During the third exposure, the stimulus played again (with the same color, location, and duration as the familiarization exposures). Whenever a response was made, a small white bar was superimposed on the progress bar to indicate the time of response. This feedback was designed to give participants cues to the distribution of their responses, which ought to increase their ability to distribute responses randomly. If participants made an incorrect number of responses, they were given an error message that reiterated the number of responses required and how many they had made. A short refractory period (200 $\mathrm{ms}$ ) between responses was hard-coded into the program, so that a single long buttonpress was not misinterpreted by the program as multiple responses, which was a necessary consideration. This refractory period made it impossible to make extremely fast sequential responses, which could feasibly be random. Notwithstanding, the number of responses in this window was expected to be liminally small, so it should not have affected the observed patterns. The refractory window might have cut the tail off subsequent modes, artificially narrowing peaks in the response distribution. However, it should not induce systematic nonrandomness into the response distribution. 


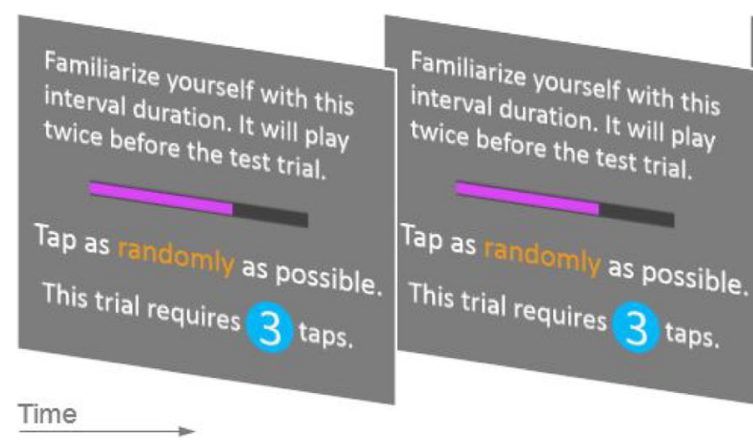

Fig. 1 Example trial time course. Participants were exposed to the temporal interval twice, by means of an animated audiovisual cue. They knew how many responses would be required in advance of the test trial.

\section{Results}

Only trials on which participants made the cued number of responses $(95.43 \%)$ were analyzed. The analysis pipeline involved three broad steps: generate normalized distributions of response times for the different conditions and instructions, and combine the data wherever appropriate to increase power; isolate the components of interest via linear detrending and smoothing; and generate bootstrapping distributions corresponding to our a priori expectations to compare against our observed data.

\section{Kolmogorov-Smirnov tests}

First, we asked whether our two instruction blocks (random or whimsical) produced different patterns of responses. For each combination of number of responses and interval duration, we compared the distributions from the two instruction conditions using a two-sample Kolmogorov-Smirnov test, which examines the difference between two distributions at every bin and determines whether the cumulative distribution functions are the same. The $\mathrm{K}-\mathrm{S}$ test failed to reject the null hypothesis that the distributions were the same for all nine comparisons. Consequently, we combined the data across instruction conditions for the subsequent analyses.

At this point, we asked whether participants were acting randomly. We conducted a two-sample $\mathrm{K}-\mathrm{S}$ test for the combined distributions at every level of number of responses and interval duration against a randomly generated (uniform) distribution of equal size. All nine tests rejected the null hypothesis (all $p \mathrm{~s}<.001$ ), confirming our expectation that participants would fail to act randomly despite the instructions.

Finally, we wanted to combine the data from trials with different interval durations, in order to increase our power in examining the effects of number of responses. To this end, we conducted pairwise two-sample $\mathrm{K}-\mathrm{S}$ tests between each combination of interval durations for each of the three levels of number of responses. All tests failed to reject the null, justifying combination of the data for the three levels of interval

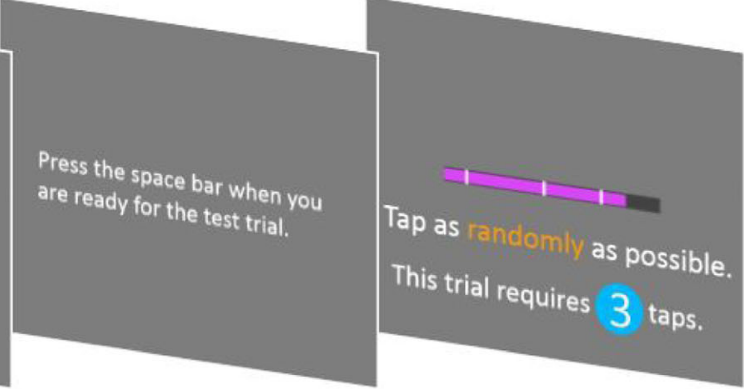

On the third exposure, the same audiovisual cue played while participants made their subdivision responses. Small white bars provided feedback to the participant, in an effort to maximize the cues for randomness

duration. In addition to increasing our power, these tests showed that any nonrandom biases in response distribution scaled to fit the maximum duration of the allowable response times. We were left with three distributions of normalized response times for trials requiring one, two, or three responses. All subsequent analyses were performed on these distributions.

\section{Distribution hygiene}

The variance in these distributions has at least two visibly discernible components. One is the local perturbations surrounding the means; this matches our a priori expectation that randomly produced responses should cluster around the whole-integer subdivisions of the interval. The second component, which appears to dominate the distributions, is a decreasing trend that is of little theoretical interest: This pattern likely corresponds to participants carefully (or impatiently) ensuring that their responses fall within the allowed duration, biasing toward earlier responses. To remove this component, we performed a linear detrending of the three distributions using only bins containing nonzero data (using bins containing zero responses at the beginning of the distribution would misrepresent the slope and intercept of the linear function). The linear detrending resulted in some negative frequencies, so the resultant distributions were normalized for frequency and bound between 0 and 1 . A one-bin sliding window smoothed the distribution by taking the average of each bin and its adjacent neighbors. After smoothing and detrending of the data, we are left with distributions representing the preserved component, which we hypothesize represents a tendency to represent intervals around their whole-integer subdivisions. These empirical distributions are depicted as the black lines in Fig. 2.

\section{Bootstrapping}

To test the hypothesis that random interval generation would result in nonrandom clusters around the whole-integer 

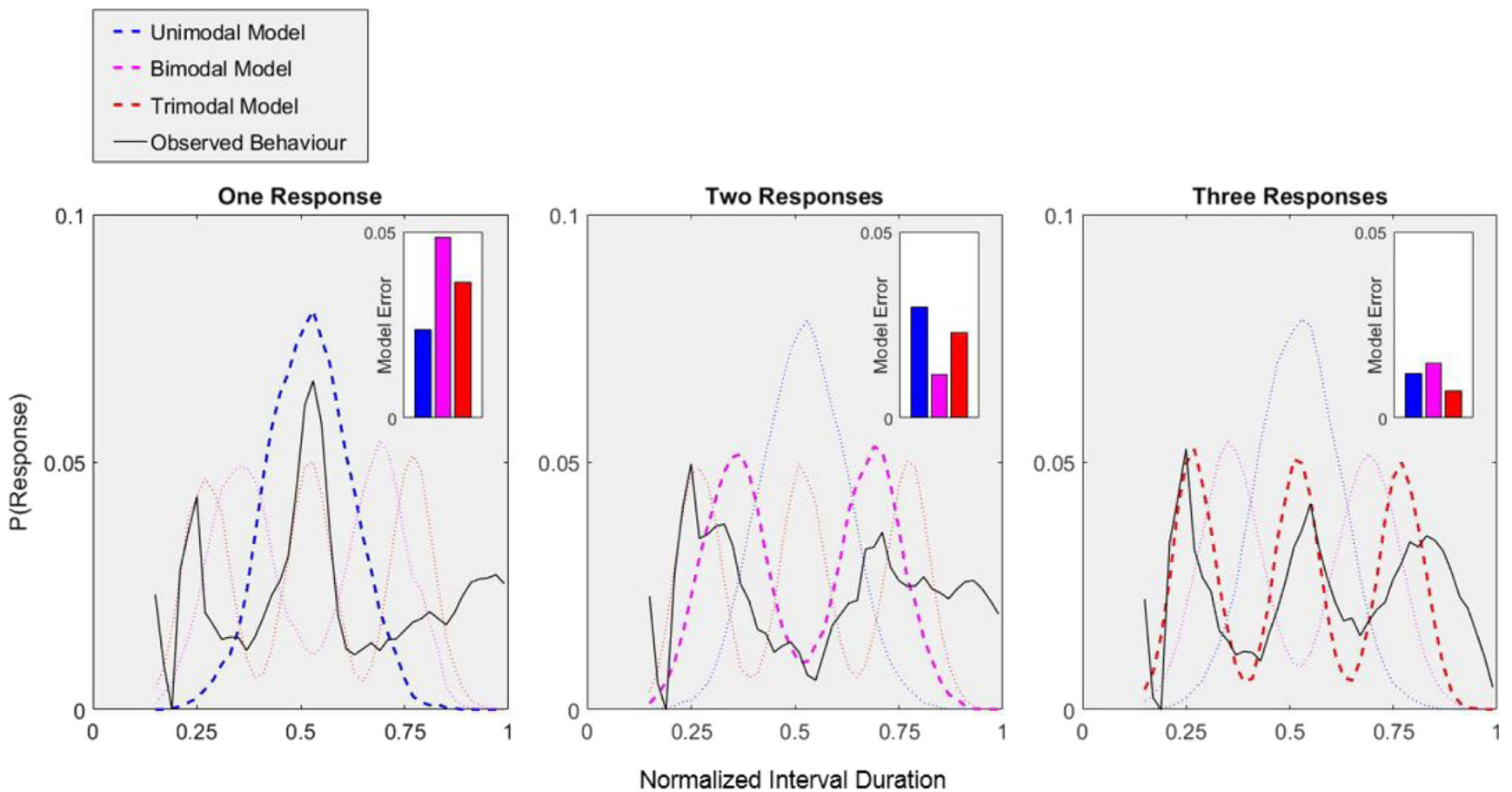

Fig. 2 Normalized empirical (black) and bootstrapping (colorful) distributions for our random-subdivision task. In each case, the bestfitting model is displayed with a thicker, dashed line. The error from the model fits is depicted in the subpanels. This analysis shows that

participants tended to subdivide the interval around $k$ equally spaced modes, where $k$ is the number of responses required. The resultant pattern is thus periodic; it approximates isochrony

subdivisions of the parent interval, we generated three bootstrapping distributions, corresponding to normalized probability distribution functions with one, two, or three modes. All three distributions were created by random sampling of the normal distribution centered on $1 / 2$ (one-response hypothesis), $1 / 3$ and $2 / 3$ (two-response hypothesis), and $1 / 4$, $1 / 2$, and $3 / 4$ (three-response hypothesis). The sample size was equal to the number of responses observed in each corresponding empirical distribution. The standard deviation of each peak was arbitrarily set according to the linearly decreasing function .125-.025k, where $k$ is the number of peaks in the bootstrapping distribution. This was done in order to account for the observation that the peaks of the multimodal distributions were blending into each other and required a narrower spread than the unimodal distribution. Note that these $S D$ values were not fitted and represent an arbitrary and highly conservative shot at estimating the nonrandom component of our data. These bootstrapping distributions were then smoothed and normalized according to the same procedure as our observed data.

These distributions are depicted in Fig. 2 in different colors for unimodal, bimodal, and trimodal distributions. In each panel, the best-fitting bootstrapping distribution is emphasized with a boldfaced dashed line. To test our hypothesis that randomly generated interval subdivisions would be nonrandomly clustered around the wholeinteger subdivisions, we calculated the sum of squares of the residuals (SSR) at each point of the three empirical distributions for one, two, or three responses against the bootstrapping distributions. The SSRs are represented in the inset panels of Fig. 2. In each case, the model with the smallest error contained the predicted number of peaks, providing evidence for our hypothesis that participants would subdivide suprasecond intervals into roughly equal subdivisions, despite instructions to act whimsically or as randomly as possible.

\section{Discussion}

We asked participants to subdivide an interval of time as randomly as possible. The results showed that participants were decidedly nonrandom, with their responses clustered around the whole-integer subdivisions of the parent interval. This conclusion was reached via a bootstrapping analysis that showed that the best-fitting model for conditions requiring $k$ random responses was described by a function with $k$ equally spaced modes. In other words, participants imposed a periodic structure onto the unstructured stimulus.

Unlike prior studies, which have shown that the perception of temporal sequences is warped toward wholeinteger ratios (e.g., Fraisse, 1946; Ravignani et al., 2016), our stimuli were nonsequential (i.e., a single duration) and 
nonstructured. Whereas a randomly generated sequence of intervals might imply structure accidentally or approximately, our intervals were whole and could not suggest a manner in which they should be divided. The results therefore imply that participants experienced a powerful bias to impose a structure upon the internally represented interval. It is important to note, however, that the present study does not identify the locus of this nonrandom imposition-whether at the representational or the response level (e.g., Firestone \& Scholl, 2015). It is alternatively possible that observers can represent random subintervals perfectly well, yet cannot produce randomly timed actions, or that each response places nonrandom constraints on the representation of the following subinterval (a reciprocal representation-production effect). Notwithstanding, the important message from this study is the shape of our nonrandomness - that time intervals are irresistibly divided by means of a simple arithmetic (interval $/ k$ responses), which dovetails perfectly with the universally human predilection for isochronous rhythm.

In separate blocks, we asked participants to perform the task either randomly or whenever they felt like it, with the expectation that different instructions might result in different patterns of structure. Instead, we found that both conditions were nonrandom, and nonrandom in the same way. Consequently, we concluded that the same bias toward a periodic structure operates identically, regardless of the task.

We contend that the irresistible periodicity of interval subdivisions reveals an easy and manageable heuristic for temporal cognition. Interestingly, there is no obvious reason why arbitrary response times should correspond to these whole-integer subdivisions, but the unconstrained nature of the task is precisely the context wherein latent, probabilistic biases in cognition should rise to the surface through action. The success of this study highlights the investigative power of methods that use random or whimsical tasks to reveal our cognitive nature.

Author note The experimental materials are available upon request. This study was not preregistered.

\section{References}

Brainard, D. H. (1997). The Psychophysics Toolbox. Spatial Vision, 10, 433-436. https://doi.org/10.1163/156856897X00357

Brugger, P. (1997). Variables that influence the generation of random sequences: An update. Perceptual and Motor Skills, 84, 627-661.

Firestone, C., \& Scholl, B. J. (2014). "Please tap the shape, anywhere you like" shape skeletons in human vision revealed by an exceedingly simple measure. Psychological Science, 25, 377-386.

Firestone, C., \& Scholl, B. J. (2015). When do ratings implicate perception versus judgment? The "overgeneralization test" for top-down effects. Visual Cognition, 23, 1217-1226. https://doi.org/10.1080/ 13506285.2016.1160171

Fraisse, P. (1946). Contribution a l'étude du rythme en tant que forme temporelle. Journal de Psychologie Normale et Pathologique, 39, 283-304.

Jacoby, N., \& McDermott, J. H. (2017). Integer ratio priors on musical rhythm revealed cross-culturally by iterated reproduction. Current Biology, 27, 359-370.

Loetscher, T., \& Brugger, P. (2007). Exploring number space by random digit generation. Experimental Brain Research, 180, 655-665.

Loetscher, T., Schwarz, U., Schubiger, M., \& Brugger, P. (2008). Head turns bias the brain's internal random generator. Current Biology, 18, R60-R62. https://doi.org/10.1016/j.cub.2007.11.015

Ravignani, A., Delgado, T., \& Kirby, S. (2016). Musical evolution in the lab exhibits rhythmic universals. Nature Human Behaviour, 1, 0007. https://doi.org/10.1038/s41562-016-0007

Repp, B. H., London, J., \& Keller, P. E. (2011). Perception-production relationships and phase correction in synchronization with twointerval rhythms. Psychological Research, 75, 227-242.

Repp, B. H., \& Penel, A. (2002). Auditory dominance in temporal processing: New evidence from synchronization with simultaneous visual and auditory sequences. Journal of Experimental Psychology: Human Perception and Performance, 28, 1085-1099. https://doi. org/10.1037/0096-1523.28.5.1085

Savage, P. E., Brown, S., Sakai, E., \& Currie, T. E. (2015). Statistical universals reveal the structures and functions of human music. Proceedings of the National Academy of Sciences, 112, 89878992. https://doi.org/10.1073/pnas.1414495112

Thompson, V. A., \& Paivio, A. (1994). Memory for pictures and sounds: Independence of auditory and visual codes. Canadian Journal of Experimental Psychology, 48, 380-398.

Vandierendonck, A. (2000). Is judgment of random time intervals biased and capacity-limited? Psychological Research, 63, 199-209.

Publisher's note Springer Nature remains neutral with regard to jurisdictional claims in published maps and institutional affiliations. 